\title{
O uso do genograma e do ecomapa na identificação de fatores de risco para a prevenção da violência doméstica contra a mulher
}

\author{
Use of genogram and ecomap in the identification of risk factors to prevent domestic \\ violence against women
}

Uso de genograma y ecomap en la identificación de factores de riesgo para la prevención de la violencia doméstica contra las mujeres

Aline Domingues Barretoํㅡㄹ Helena Carolina Viana Alves ${ }^{1}$, Jéssica Brambati Martins ${ }^{1}$, Julia de Alcântara Moura ${ }^{1}$, Laura Mariana Vieira Correa ${ }^{1 *}$, Natália Bianca Vales Bhering ${ }^{1}$, Verônica de Araújo Libânio Costa ${ }^{1}$, Thereza Cristina Gomes Horta² .

\section{RESUMO}

Objetivo: Analisar o uso do genograma e do ecomapa como ferramentas da Medicina de Família e Comunidade (MFC) na identificação e prevenção da Violência Doméstica Contra a Mulher (VDCM). Revisão bibliográfica: Com a pandemia por COVID-19, a VDCM que já era tida como grave problema de saúde pública, acentuou-se ainda mais. Devido a recorrência de fatores de risco na história das vítimas, a identificação desse problema de saúde na Atenção Primária à Saúde (APS) torna-se possível e oportuna. O MFC, como profissional inserido na APS, deve se dispor à escuta atenta da história e das vivências pessoais da paciente, para que a identificação desses fatores de risco se concretize. Nesse contexto, as ferramentas genograma e ecomapa apresentam-se como possibilidades terapêuticas promissoras na investigação clínica da VDCM, relevantes por seu potencial de identificar os fatores de risco no contexto familiar e compreender as redes de apoio familiar e social às quais a vítima pode recorrer. Considerações finais: Reforça-se a relevância do uso do genograma e ecomapa na identificação e prevenção da VDCM pelo MFC inserido na APS.

Palavras-chave: Atenção primária à saúde, Medicina de família e comunidade, Violência doméstica.

\begin{abstract}
Objective: To analyze the use of the genogram and the ecomap as tools of Family and Community Medicine (FCM) in the identification and prevention of Domestic Violence Against Women (DVAW). Bibliographic review: In the COVID-19 pandemic, the DVAW which was already considered a serious public health problem, became even worse. Due to the recurrence of certain risk factors in the victims' history, the identification of this health problem in Primary Health Care (PHC) becomes possible and opportune. The FCM, as a professional inserted in the PHC, must be willing to listen carefully to the patient's history and personal's experiences so the identification of these risk factors can be effective. In this context, the genogram and ecomap tools are promising therapeutic possibilities in the clinical investigation of DVAW, relevant for their potential to identify risk factors in the family context and to understand the family and social support networks to which the victim can resort. Final considerations: The relevance of using the genogram and ecomap in the identification and prevention of DVAW by the FCM inserted in PHC is reinforced.
\end{abstract}

Keywords: Primary health care, Family practice, Domestic violence.

\footnotetext{
${ }^{1}$ Pontifícia Universidade Católica de Minas Gerais (PUC-MG), Betim - MG.

*E-mail: lauravieira08@gmail.com

2 Centro Universitário Uni BH, Belo Horizonte - MG.
} 


\section{RESUMEN}

Objetivo: Analizar el uso del genograma y el ecomap como herramientas de medicina familiar y comunitaria (MFC) en la identificación y prevención de la violencia doméstica contra la mujer (VDCM). Revisión bibliográfica: Con la pandemia de COVID-19, la VDCM que ya se consideraba un problema grave de salud pública, empeoró aún más. Debido a la recurrencia de ciertos factores de riesgo en el historial de las víctimas, la identificación de este problema de salud en Atención Primaria de Salud (APS) se hace posible y oportuna. EI MFC, como profesional insertado en la APS, debe estar dispuesto a escuchar atentamente el historial del paciente y sus experiencias personales para que la identificación de estos factores de riesgo sea efectiva. En este contexto, las herramientas de genograma y ecomap son posibilidades terapéuticas prometedoras en la investigación clínica de la VDCM, relevantes por su potencial para identificar factores de riesgo en el contexto familiar y comprender las redes familiares y de apoyo social a las que la víctima puede recurrir. Consideraciones finales: Se refuerza la relevancia de usar el genograma y el ecomap en la identificación y prevención de la VDCM por el MFC insertado en APS.

Palabras clave: Atención primaria de salud, Medicina familiar y comunitaria, Violencia doméstica.

\section{INTRODUÇÃO}

A violência doméstica contra a mulher (VDCM) é um fenômeno sociocultural que resulta de relações baseadas em poder e conflito entre gêneros (SANTOS WJ, et al., 2018). Pela Lei $n^{0} 11.340$, de 7 de agosto de 2006, é definida como

"Qualquer ação ou omissão baseada no gênero que the cause morte, lesão, sofrimento físico, sexual ou psicológico e dano moral ou patrimonial: no âmbito da unidade doméstica, [...] no âmbito da família, [...] em qualquer relação íntima de afeto, independentemente de coabitação" (BRASIL, 2006).

Essa violência tem grande importância, haja visto as graves consequências para as vítimas e para a sociedade, que incluem até mesmo morte, além da alta prevalência e da tendência de crescimento (SANTOS AC, et al., 2018). O assunto se torna ainda mais relevante no contexto atual, com aumento no número de casos resultante do período de isolamento social essencial para o controle da pandemia por Coronavírus (COVID-19) (VIEIRA PS, et al., 2020).

A identificação dos fatores de risco para a VDCM é fundamental para a prevenção efetiva de sua ocorrência. Segundo o Ministério da Saúde (MS), os serviços de Atenção Primária à Saúde (APS) são estratégicos para isso devido a reorganização do modelo assistencial, por meio da Estratégia Saúde da Família (ESF). No entanto, na prática, é possível observar que os profissionais atuantes na APS, incluindo os médicos, ainda apresentam grande dificuldade na abordagem correta da VDCM, não cumprindo de maneira totalmente eficaz seu papel (SANTOS AC, et al., 2018; SANTOS WJ, et al., 2018; HOLANDA ER, et al., 2018).

Sendo assim, é necessário a apropriação de saberes capazes de possibilitar a prevenção da VDCM no contexto da APS (SANTOS AC, et al., 2018). Para esse propósito, o genograma e o ecomapa são instrumentos úteis criados por terapeutas familiares e utilizados na prática da Medicina de Família e Comunidade (MFC), que têm por objetivo avaliar e proporcionar aos profissionais a perceptibilidade da composição familiar, além de aprofundar nas relações existentes entre o paciente e o meio social em que vive (BARRETO M e CREPALDI MA, 2017).

Este artigo trata-se de uma revisão narrativa de literatura com abordagem crítica e integrativa, com intuito de construir, atualizar o conhecimento e incorporá-lo na prática clínica e social. O delineamento adotado de pesquisa bibliográfica permite ao investigador uma abrangência muito ampla de informações, o que fornece um bom aparato para a discussão do problema apresentado e viabiliza a integração dos conhecimentos científicos da temática, contribuindo assim, para o desenvolvimento de novos aprendizados (GIL AC, 1999; CRESWELL JW, 2010). A revisão de literatura garante não apenas um resumo dos estudos, mas também uma avaliação de seus pontos positivos e negativos, a fim de estabelecer uma base para as questões levantadas e para as hipóteses que surgirem (CRESWELL JW, 2010).

Portanto, o objetivo desta revisão é analisar o uso das ferramentas terapêuticas genograma e ecomapa, amplamente utilizadas pela MFC, na identificação dos fatores de risco e na prevenção de VDCM. 


\section{REVISÃO BIBLIOGRÁFICA}

Segundo Vieira PS et al. (2020), a prevenção da VDCM é um desafio a ser enfrentado na sociedade, visto que, na prática, a garantia de segurança e de descanso no lar é vista como um privilégio da classe e do gênero. A perpetuação da VDCM, então, deve ser relacionada ao machismo estrutural, pelo qual o homem é compreendido como sendo a figura mais forte e mais competente nas relações sociais, física e psicologicamente, quando comparado à mulher. A lógica patriarcal também é um componente da problemática em questão, pois tenta legitimar essa imagem masculina como chefe da família (SANTOS ACW e MORÉ CLOO, 2011).

\section{Epidemiologia da VDCM}

Conforme dados fornecidos pelas Delegacias Especializadas no Atendimento às Mulheres (DEAM), 205 mil agressões a mulheres são notificadas por ano no país. Ademais, o número de vítimas de homicídio do sexo feminino aumentou em $21,0 \%$ nos últimos 10 anos, segundo o Sistema de Informação de Agravos de Notificação (Sinan) (SANTOS WJ, et al., 2018). Nesse cenário apresentado, a VDCM tornou-se a principal causa de morte e de invalidez entre mulheres.

No Brasil, essa situação se agravou com o isolamento social referente à pandemia enfrentada da COVID19, em função da exacerbação de fatores, a exemplo da coexistência forçada de agressores e vítimas, do estresse econômico e do aumento do consumo de bebidas alcoólicas (VIEIRA PS, et al., 2020). De acordo com o levantamento realizado pela Ouvidoria Nacional dos Direitos Humanos (ONDH), do Ministério da Mulher, da Família e dos Direitos Humanos (MMFDH) houve um aumento de 18\% nas denúncias de violência em março deste ano. Em acréscimo, apresentou-se que $88,8 \%$ dos feminicídios notificados foram executados por companheiros ou ex-companheiros das vítimas (VIEIRA PS, et al., 2020).

\section{Tipos de VDCM e suas consequências}

É fundamental que o médico e os integrantes da equipe de saúde reconheçam os sinais da violência contra a mulher. Para isso, é necessário saber as formas de maus tratos que existem, sendo elas: abuso emocional/psicológico (qualquer conduta que resulte em dano emocional como, a diminuição da autoestima, coação, humilhações, imposições, jogos de poder, desvalorização, xingamentos, gritos, desprezo, desrespeito, entre outras transgressões dos valores morais); abuso físico (o uso da força física ou de alguma outra arma provoca danos não acidentais e lesões corporais; exemplificada por tapas, empurrões, perfurações, chutes, murros, queimaduras, tiros, etc.); violência patrimonial (destruição de bens materiais, objetos e documentos da vítima); abuso sexual (ação em que uma pessoa em relação de poder e pelo uso de força física, coerção ou intimidação psicológica, obriga a outra ao ato sexual contra sua vontade, ou que a exponha em interações sexuais que propiciem sua vitimização, da qual o agressor tenta obter gratificação) (FONSECA DH, et al., 2012).

Ressalta-se que, em consequência da VDCM, as mulheres vivenciam diversos traumas nos âmbitos físico, mental, emocional e espiritual, que podem coexistir. Dentre os problemas de saúde mais frequentes apresentados por elas, encontram-se queixas de dores severas, dificuldades de concentração, tontura, tentativa de autoextermínio e maior frequência do consumo de bebidas alcoólicas.

Considerando que os atos de violência não ocorrem de maneira isolada, mas persistem por anos, as vítimas também estão sujeitas a maiores riscos de desenvolver transtornos psicopatológicos, como o transtorno de estresse pós-traumático (TEPT), a síndrome da dor crônica, as dificuldades de socialização e, também, os problemas referentes à saúde reprodutiva (HOLANDA ER, et al., 2018). Em acréscimo, essa situação acarreta aumentos nos custos econômicos para o país, por meio de gastos com o suporte médico em saúde ou pela diminuição da produtividade laboral da vítima, como exemplos (SANTOS WJ, et al., 2018; TONDOWSKI CS, et al., 2014; SANTOS AC, et al., 2018).

\section{A importância da Atenção Primária em Saúde e da Medicina de Família e Comunidade na prevenção da VDCM}

As ações preventivas, de acordo com Cavalcanti GMB et al. (2020), visam à diminuição de riscos contra a integridade física e moral da mulher, à garantia de proteção no lar - local onde espera-se o cuidado entre os habitantes-, e ainda, evitam a banalização e a perpetuação desse tipo de violência na sociedade. É nesse cenário, onde existem fatores de risco associados à violência doméstica, que a APS, dando ênfase no papel do médico de família e comunidade, deve atuar na perspectiva preventiva. 
A Medicina de Família e Comunidade (MFC) é uma especialidade que presta assistência à saúde de maneira integral, continuada e em todos os ciclos da vida para o paciente, sua família e comunidade no qual ele está inserido. Ela perpassa a clínica tradicional ao usar o método clínico centrado na pessoa, a clínica ampliada e compartilhada, a abordagem familiar e coletiva territorial. A função desses profissionais vai além das ações curativas, eles são responsáveis pela promoção da saúde, prevenção de doenças e agravos, acompanhamento longitudinal e cuidados paliativos. Para cumprir seu papel, podem ser empregadas diversas ferramentas que são próprias da especialidade de acordo com a necessidade da abordagem (ANDRADE HS, et al., 2018).

Esses profissionais estão em posição estratégica na abordagem e na prevenção da VDCM, uma vez que a organização desse modelo assistencial oportuniza espaços para denúncias, para assistência e para proteção da paciente vítima de violência. Em adição, no que diz respeito à prevenção, as características desse serviço, como a instituição de vínculo de confiança com as usuárias e a longitudinalidade do acompanhamento integral, são muito importantes e devem ser exploradas nesse contexto (SANTOS AC, et al., 2018).

Diante do exposto, compreende-se a complexidade do atendimento às vítimas de violência doméstica, as quais isolam-se dos seus contatos sociais, mantendo-se restritas ao ambiente doméstico e, consequentemente, afastando-se da sua rede de apoio. Esse panorama as torna mais encarceradas no ciclo de agressões, sejam elas físicas, verbais, sexuais ou psicológicas (SANTOS ACW e MORÉ CLOO, 2011).

O profissional da APS responsável por receber esta paciente, segundo Silva AS et al. (2015), precisa criar meios para identificar condições predisponentes da VDCM e sintetizar artifícios para que os seus valores culturais e morais não interfiram no atendimento. Para que esse objetivo seja alcançado, é necessário oferecer um acolhimento adequado e que garanta um ambiente no qual a mulher possa sentir-se confortável. A capacidade de uma escuta sem julgamentos é inerente a esse processo, e deve ser cercada de sigilo profissional. Ainda assim, o ouvinte pode se deparar com a dificuldade da mulher em afrouxar as fronteiras da família e em admitir o seu sofrimento, seja por medo, vergonha e sentimento de culpa, resultantes da cultura machista em vigência.

\section{O papel do genograma e do ecomapa na identificação dos fatores de riscos e na prevenção da VDCM}

É necessário valer-se de conhecimentos e de ferramentas que permitam a prevenção da VDCM na APS de forma coerente e aplicável na prática clínica (SANTOS AS, et al., 2018). Nessa abordagem, o genograma e o ecomapa são instrumentos válidos empregados para a análise do indivíduo no contexto familiar, de maneira a tentar identificar o todo de sua rede de apoio social e familiar (NASCIMENTO LC, et al.; 2014). Por intermédio destes, pode-se efetivar uma mudança paradigmática da visão centrada no indivíduo para uma visão do sistema relacional, portanto, dentro de um contexto vinculado ao seu ambiente (MUNIZ JR e EISENTEIN E, 2009).

É de extrema importância identificar a constituição familiar da vítima, uma vez que os fatores de risco estão presentes, principalmente, no ambiente doméstico. Na prática clínica diária, um método apropriado e que pode ser empregado é a construção do genograma familiar. Ele é um instrumento de avaliação familiar utilizado na MFC, que consiste em um sistema de coleta e de registro de dados, por meio de um fluxograma, com o objetivo de relacionar a história biomédica e psicossocial da paciente com a de sua família, a fim de ser utilizado como base de análise e de mecanismo de diálogo (MACHADO RR, et al., 2015). Para uma melhor compreensão da funcionalidade prática do genograma, faz-se necessário exemplificar, utilizando-se de recursos de imagem, o significado dos traçados e símbolos constituintes deste método de análise.

Dentre as inúmeras possibilidades, é possível fazer a diferenciação simbólica de gêneros, identificar a data de nascimento e a idade, representar casamentos utilizando-se de traçados, assim como inserir a data da união, e ainda, identificar a presença de separação conjugal, de divórcio, e daqueles que voltaram a morar juntos. Os filhos são representados na segunda geração em ordem de nascimento a partir da esquerda, tendose a possibilidade de desenhar a existência de filhos adotados ou de criação. Além dos símbolos, existem as linhas de moradia, e de relacionamento, que auxiliam na identificação dos moradores e dos laços existentes entre eles. Esses traçados podem significar relacionamento ligado, rompido, fusionado, conflituoso, distante, muito ligado com conflito e a presença de abuso físico, abuso emocional e abuso sexual (BARRETO $\mathrm{M} \mathrm{e}$ CREPALDI MA, 2017). 
Razera J e Falcke D (2014) também consideram a violência conjugal como um fenômeno complexo, que demanda ampla investigação. Por isso, visando uma análise mais completa e uma melhor compreensão do contexto familiar de um casal estudado, as autoras utilizaram, dentre outros instrumentos, o genograma familiar.

Essa ferramenta permitiu visualizar as dinâmicas e os vínculos das famílias de origem e da família nuclear. A partir do genograma construído em suas análises, foi possível observar que tanto as relações conjugais, quanto as existentes entre pais e filhos eram marcadas pela violência, como observado por meio da simbologia padronizada dos traçados.

Apesar de essa técnica não especificar o tipo de violência retratado, o estudo demonstrou que foi possível aferir que a violência conjugal da família nuclear era baseada em agressões psicológicas por parte da esposa e em coerção sexual por parte do marido.

É possível observar, ainda, a transgeracionalidade da VDCM no contexto familiar, mesmo que manifestada de formas variadas, visto que os cônjuges tendem a repetir padrões de relacionamentos experienciados nas suas famílias de origem.

Além disso, fatores de risco a serem abordados, como o consumo excessivo de álcool e possíveis consequências psicopatológicas da vivência de um relacionamento abusivo e violento, como a depressão, podem ser identificados em via de análise.

Em adição, uma vez que a VDCM é longitudinal, a detecção precoce de relações conflituosas e desarmônicas permitem intervenção prévia por parte da equipe de saúde e previnem a perpetuação da violência (RAZERA J e FALCKE D, 2014). (Figura 1 e 2).

Figura 1 - Genograma Familiar.

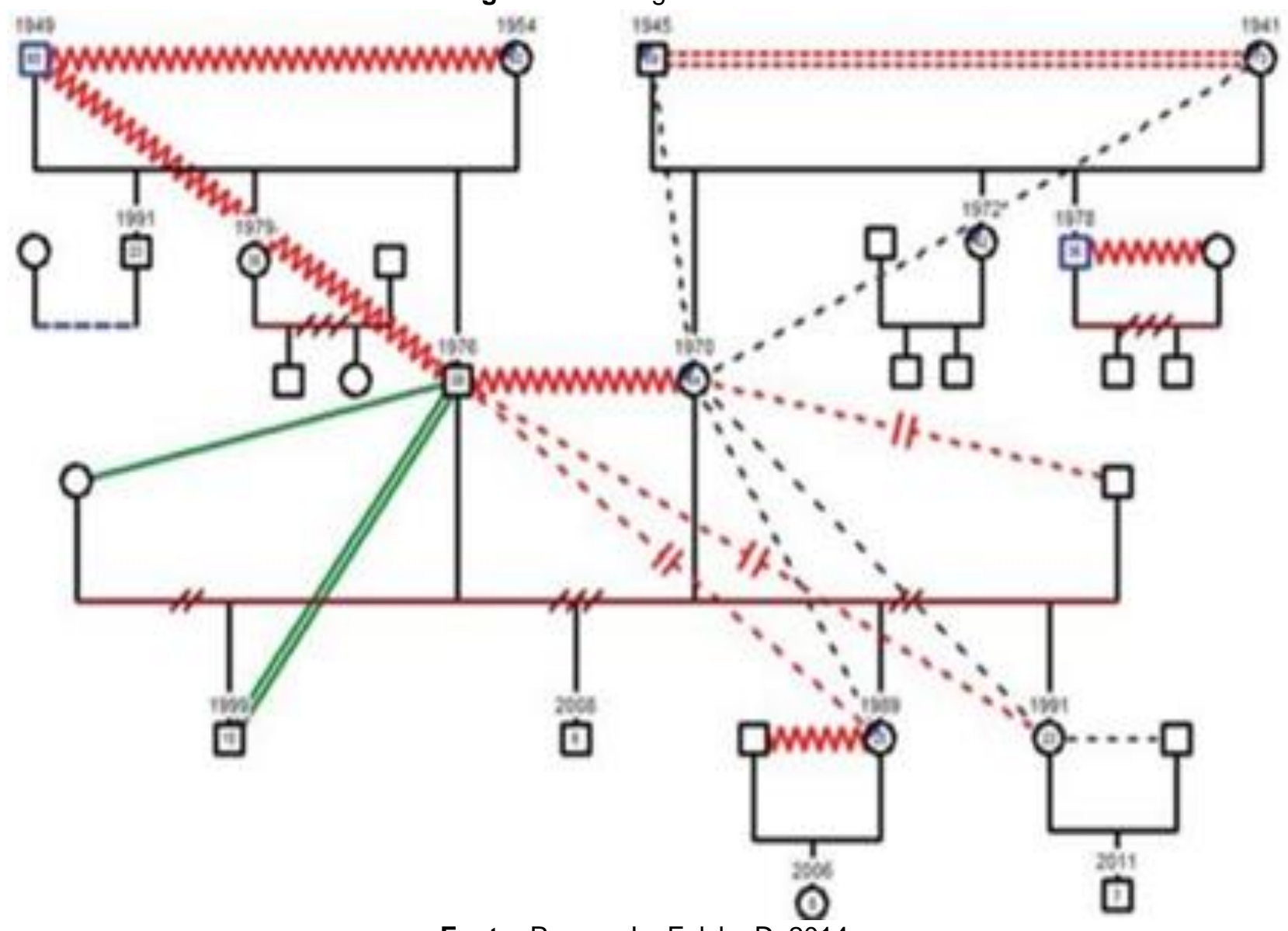

Fonte: Razera J e Falcke D, 2014. 
Figura 2 - Legenda do Genograma Familiar.

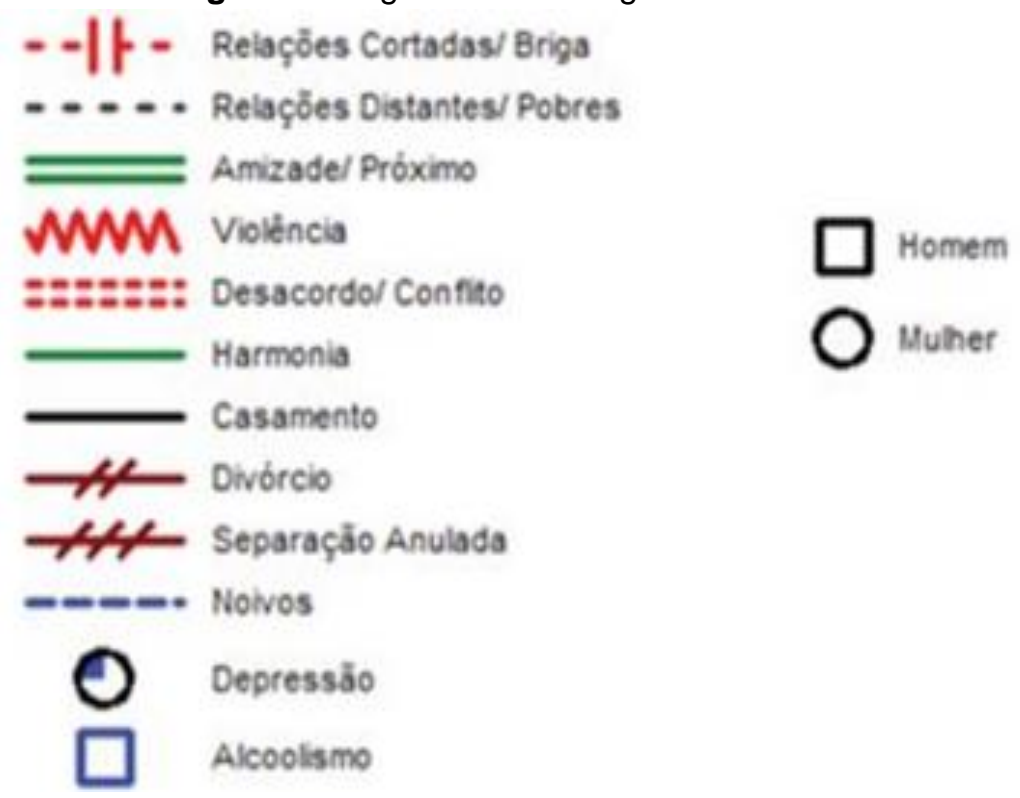

Fonte: Razera J e Falcke D, 2014.

O ecomapa, por sua vez, é definido como um diagrama das relações entre a família e a comunidade, que desenha o sistema ecológico onde a família ou o indivíduo está inserido, reconhecendo seus modelos de organização e a natureza das suas relações com o meio onde habita, indicando o balanço ou, no caso da VDCM, o desequilíbrio entre os seus recursos e as suas necessidades (BOTTI NCL, et al.; 2013). Possibilita, então, uma visão mais ampliada, focalizada no presente, dos relacionamentos em análise com os outros sistemas, como escola, trabalho e amizades (MACHADO RR, et al., 2015).

Além da identificação dos fatores de risco e a possibilidade de atuação nestes, a fim de evitar que os episódios violentos ocorram, esses instrumentos permitem avaliar se a vítima em atenção tem uma rede de apoio familiar e social eficaz e presente, qual a composição desta ou os motivos que impedem a sua existência. Essas redes de apoio são essenciais na prevenção e no suporte às mulheres que desejam romper os círculos violentos. Um indivíduo com poucas conexões com a comunidade e com seus membros, tem maior risco de sofrer violência, por isso necessita de um maior investimento da equipe de saúde. É válido lembrar que a família é o apoio social mais consistente do sujeito e que existe uma explícita interdependência entre seus componentes, a qual deve ser saudável, sem excessos (MACHADO RR, et al., 2015).

Dentro desse contexto, diversos autores referem que ao analisar genogramas familiares em que a VDCM estava presente, foi possível apontar inúmeros fatores comuns como causas recorrentes, tais como a presença de alcoolismo e existência de conflitos familiares, tanto em gerações anteriores, quanto na família em investigação, comportamento agressivo do marido, presença de fatores socioeconômicos como desemprego, dificuldade em revelar insatisfações para com a parceira, ausência de negociação de conflitos conjugais e a observação de violência psicológica e física constante entre o casal (COLOSSI PM e FALCKE D, 2013; SANT'ANNA TC e PENSO MA, 2017). Essas informações, consequentemente, permitem antecipar potenciais problemas familiares e/ou identificar os atuais, que podem se referir à instauração ou ao agravamento de situações de VDCM.

Voltando-se às vítimas da violência, comumente são mulheres jovens, de baixos níveis de apoio social, de baixa escolaridade, em união estável e com condições econômicas precárias. Essas características, dentre outras, podem ser coletadas por meio da elaboração do genograma e do ecomapa, a partir da escuta atenciosa de sua história de vida e vivências pessoais, por exemplo. A dependência financeira ou emocional para com o companheiro também constitui um fator a ser investigado, tendo em vista que essas mulheres apresentam maior dificuldade em romper a relação por se sentirem subordinadas ao agressor (SANTOS WJ, et al., 2018; HOLANDA ER, et al., 2018). 
Portanto, ambas as ferramentas se complementam para uma abordagem mais integral da paciente, podendo ser considerados recursos organizadores e clarificadores de grande utilidade no trabalho terapêutico por parte do médico. Sendo assim, elas são relevantes na identificação de fatores de risco, formulação de hipóteses e atuação da equipe de saúde de forma mais dirigida e eficiente (BARRETO M e CREPALDI MA, 2017).

Apesar da VDCM constituir na atualidade um grave problema de saúde pública, esse assunto é diversas vezes negligenciado pelos profissionais de saúde da APS, principalmente quando não existem lesões físicas visíveis, reforçando a importância da atuação do MFC e do uso do ecomapa e do genograma (SOUZA AAC e CINTRA RB, 2018). Também é possível haver um insuficiente domínio de conceitos legais, levando o terapeuta a agir de acordo apenas com seus conhecimentos de senso comum (LEITE AC e FONTANELLA BJB, 2019).

Nesse sentido, a implementação das ESF organizadas em rede torna-se fundamental na notificação, no manejo e no tratamento dos casos de violência (CAVALCANTI GMB, et al., 2020). É válido afirmar que a violência contra a mulher é um caso de notificação compulsória de caráter sigiloso tanto nos serviços de saúde públicos quanto privados, portanto cabe também ao médico de família e comunidade notificar o ocorrido (LEITE AC e FONTANELLA BJB, 2019).

O profissional da saúde, ao entrar na vida de um paciente, torna-se responsável pelo seu cuidado; de forma que ele necessita procurar meios e instrumentos que permitam a mulher ter autonomia e empoderamento para construir sua saúde - no conceito mais amplo da palavra (ZUCHI ZC, et al., 2018). Para que esse modelo de assistência seja efetivo, é preciso que o médico tenha empatia e conheça os aspectos socioeconômicos, culturais e históricos dessas pacientes.

Assim, ele será capaz de compreender de maneira integral a funcionalidade da família e da comunidade em que a mulher está inserida e estabelecer um plano terapêutico ideal e executável. Diante disso, a atenção do MFC aos possíveis sinais e sintomas da usuária, associada à clínica ampliada e ao uso dessas ferramentas, pode diminuir a invisibilidade das agressões presentes e daquelas em potencial (CAVALCANTI GMB, et al., 2020).

Cabe à Medicina, especialmente à MFC, então, se ocupar não apenas dos sofrimentos ligados às doenças biológicas, mas também dos preconceitos em relação às pessoas e aos comportamentos, fontes geradoras também de sofrimento. É necessário que o médico saiba abordar a violência, mesmo que essa não seja a queixa principal ou o objetivo da consulta. Muitas vezes, o profissional precisa escutar e buscar identificar os possíveis fatores de risco, a fim de garantir o cuidado integral à mulher usuária da APS (BRASIL MAA, et al., 2012).

\section{CONSIDERAÇÕES FINAIS}

Diante do exposto, reforça-se a importância das ferramentas genograma e ecomapa como linhas terapêuticas a serem utilizadas na APS para a identificação e a prevenção da VDCM, tendo em vista o aumento de casos de violência a cada ano e seu agravo recente pela pandemia por COVID-19, o que demonstra a relevância e a atualidade do tema. O genograma permite entender o contexto familiar e, assim, identificar os fatores de risco para o problema de saúde em questão. O ecomapa, por sua vez, é útil no reconhecimento das redes de apoio social e familiar às quais a vítima pode recorrer. O Médico de Família e Comunidade, como profissional inserido na APS, tem a longitudinalidade do acompanhamento integralizado e o vínculo com as pacientes a seu favor, além de possuir conhecimento acadêmico prévio a respeito do uso das ferramentas em análise, o que propicia a aplicabilidade clínica eficaz na identificação de sinais e na prevenção desse importante problema de saúde pública. A prevenção, a partir de tais instrumentos, torna-se ainda mais necessária quando não existem danos físicos, sendo fundamental para o estabelecimento de uma terapêutica adequada e possível de ser implantada. Portanto, a antecipação de potenciais fatores de risco é essencial na redução da incidência e do agravamento da VDCM. 


\section{REFERÊNCIAS}

1. ANDRADE HS, et al. A formação discursiva da Medicina de Família e Comunidade no Brasil. Physis: Revista de Saúde Coletiva, 2018; 28 (3): 1-18.

2. BARRETO M, CREPALDI MA. Genograma no contexto do SUS e SUAS a partir de um estudo de caso. Nova Perspectiva Sistêmica, 2017; 58: 74-85.

3. BOTTI NCL, et al. Ecomapa e apgar familiar na atenção à família com portador de transtorno mental. Revista APS, 2012; 15(3): 277-286.

4. BRASIL. Diário Oficial da União. Lei no. 11.340, de 7 de agosto de 2006. Dispõe sobre a violência doméstica contra a mulher. Brasília, 2006.

5. BRASIL MAA, et al. Psicologia Médica: a Dimensão Psicossocial da Prática Médica. Rio de Janeiro: Guanabara Koogan, 2012; 182p.

6. CAVALCANTI GMB, et al. A violência contra a mulher no sistema único de saúde. R. pesq.: cuid. fundam. online, 2020; 12: 146-154.

7. COLOSSI PM, FALCKE D. Gritos do Silêncio: A Violência Psicológica no Casal. Psico, $2013 ; 44$ (3): $310-318$.

8. CRESWELL JW. Projeto de pesquisa métodos qualitativo, quantitativo e misto. 3. ed. Porto Alegre: Artmed, 2010.

9. FONSECA DH, et al. Violência doméstica contra a mulher: realidades e representações sociais, $2012 ; 24$ (2), $307-$ 314.

10. GIL AC. Métodos e técnicas de Pesquisa Social. 5. ed. São Paulo: Atlas, 1999.

11. HOLANDA ER, et al. Fatores associados à violência contra as mulheres na atenção primária de saúde. Revista Brasileira em Promoção da Saúde, 2018; 31(1): 1-9.

12. LEITE AC, FONTANELLA BJB. Violência doméstica contra a mulher e os profissionais da APS: predisposição para abordagem e dificuldades com a notificação. Revista Brasileira de Medicina de Família e Comunidade, 2019; 14: 2059.

13. MACHADO RR, et al. O genograma e o ecomapa como instrumentos para compreender a rede familiar de uma pessoa com transtorno mental: um estudo de caso. Caçador, 2015; 4(2): 154-168.

14. MUNIZ JR e EISENTEIN E. Genograma: informações sobre família na (in)formação médica. Revista Brasileira de Educação Médica, 2009; 33(1): 72-79.

15. NASCIMENTO LC, et al. Genograma e ecomapa: contribuições da enfermagem brasileira. Texto Contexto Enferm, $2014 ; 23(1): 211-20$.

16. RAZERA J, FALCKE D. Relacionamento conjugal e violência: sair é mais difícil que ficar? Aletheia, 2014; 45: 156167.

17. SANT'ANNA TC, PENSO MA. A Transmissão Geracional da Violência na Relação Conjugal. Psicologia: Teoria e Pesquisa, 2017; $33(3): 1-11$.

18. SANTOS AC, et al. Violência Contra a Mulher: como os profissionais na atenção primária à saúde estão enfrentando esta realidade? Saúde e Pesquisa, 2018; 11(2): 359-368.

19. SANTOS ACW, MORÉ CLOO. Impacto da violência no sistema familiar de mulheres vítimas de agressão. Psicologia: Ciência e Profissão, 2011; 31(2): 220-235.

20. SANTOS WJ, et al. Violência Doméstica Contra a Mulher Perpetrada por Parceiro Íntimo: Representações Sociais de Profissionais da Atenção Primária à Saúde. Revista Fundamental Care Online, 2018 jul./set.; 10(3):770-777.

21. SILVA AS, et al. Análise da Violência Doméstica na Saúde das Mulheres. Journal of Human Growth and Development, 2015; 25(2): 182-186.

22. SOUZA AAC, CINTRA, RB. Conflitos éticos e limitações do atendimento médico à mulher vítima de violência de gênero. Revista bioética, 2018; 26 (1): 77-86.

23. TONDOWSKI CS, et al. Padrões intergeracionais de violência familiar associada ao abuso de bebidas alcoólicas: um estudo baseado em genogramas. Psicologia: Reflexão e Crítica, 2014; 27(4): 806-814.

24. VIEIRA PS, et al. Isolamento social e o aumento da violência doméstica: o que isso nos revela? Revista Brasileira de Epidemiologia, 2020; 23: 1 - 5.

25. ZUCHI CZ, et al. Violência contra as mulheres: concepções de profissionais da estratégia de saúde da família acerca da escuta, 2018; 22: 1085. 